\title{
Simulation based Evaluation of a Simple Channel Distribution Scheme for MANETs
}

\author{
Muhammad Asif ${ }^{1}$, Ghani-ur-rehman ${ }^{2}$ Israrullah $^{3}$ \\ ${ }^{1}$ (Computer Science, International Islamic University, Islamabad, Pakistan) \\ ${ }^{2}$ (Computer Science, Khushal Khan Khattak University Karak, Pakistan) \\ ${ }_{3}^{3}$ (Computer Science, National University of Computer\& Emerging Sciences, Pakistan)
}

\begin{abstract}
Mobile Ad-hoc Networks (MANETs) are special kind of wireless networks with no centralized control. They are ideal candidate for several real world applications where network need to be established on the fly e.g. disaster hit areas, hospitals, military surveillance etc. Researchers have been actively working in this area for a decade or so to make this concept a reality. MANETs differ from conventional fixed networks and therefore pose several challenges to the researchers working in the area. Among many others, access to shared medium is the major problem in MANETs. In such networks MAC layer protocol plays an important role in efficient utilization of shared media in distributed fashion. At the MAC layer we have three contradicting requirements, Maximize channel utilization, Minimize Control overhead and Fairness. Multi-Channel MAC protocols are considered is the most suitable solution to achieve the desired objectives but channel assignment and reservation in a distributed fashion is still a big challenge. In this paper, we have proposed a MultiChannel scheme to address the problem of channel assignment the proposed scheme is Node id based. The proposed Scheme has been compared with conventional single channel scheme using ns-2 simulation and results shows that the proposed technique gives better performance.
\end{abstract}

Keywords- BEB algorithm, Clear to send, Control channels, Dedicated Channels, MAC, Overhead, and request to send.

\section{Introduction}

The ad hoc networks are consists of mobile nodes that exchange packets by sharing a common broadcast radio channel [1], [2]. The bandwidth available for communication to be shared among the nodes is limited, therefore, it is desirable that access to this shared medium should be controlled in a way that can utilize the bandwidth efficiently, and guarantee fairness to all nodes. Thus design of a media access control (MAC) protocol is challenging problem for ad hoc network [2], [3]. In the MANETs nodes have limited resources in term of power, energy and processing power. In such networks MAC layer protocol plays an important role in efficient utilization of shared media in distributed fashion. At the MAC layer we have three contradicting requirements.

○ Maximize channel utilization.

○ Minimize Control overhead

- Ensure Fairness

Access to this shared medium should be controlled in such a way that all nodes should receive a fair share of the available bandwidth with minimum control overhead and optimal (overall) bandwidth utilization. After studying various MAC Multi channel protocols [4], [5], [6], [7], we come to the conclusion that the time synchronization among nodes for channel reservation creates control overhead. Some of these protocols suggest the usage of a dedicated channel known as control channel. The idea of control channel helps in segregating control and data packets but at the same time, it is also considered as wastage of bandwidth in a lightly congested scenario. Similarly, fairness and energy expenditure results are also dependent upon efficiently usage of control channel. In this paper, we present an idea of simple multi-channel MAC protocol where nodes are assigned a fixed channel for transmission based on their IDs which helps in avoiding the control overhead. As a result of this scheme, we have the possibility of having neighboring nodes assigned same channel for transmission but as the nodes are randomly distributed therefore the probability of having such active neighboring nodes is very low and have negligible impact on performance and the same is confirmed from results.

The rest of the paper is organized as follows: the related work is presented in Section II. Proposed scheme is presented in Section III. Empirical evolution is discussed in section IV. Performance evaluation results are discussed in Section V. We conclude the paper in Section VI. 


\section{Related Works}

We have studied the various types of Single and Multi channel MAC protocols. A brief review of some protocols that are closely related to our work is presented here. In the CSMA [2], when a sender want to send some data, it first sense the channel for the carrier data, if the carrier is present then it will try after some random period of time. If no carrier is present then it transmits data. CSMA sense the state of channel only at the transmitter. So CSMA does not overcome the hidden terminal problem. But in the ad hoc wireless networks, the sender and receiver may not be near each other all times. In such situations the packets transmitted by node are prone to collision at the receiver due to simultaneous transmissions by hidden terminals. Because both the senders are not in direct range so they both transmit at the same time and the collision will occur at the receiver side. The bandwidth utilization in CSMA protocol is less because of the exposed terminal problem. The Media Access Control Avoidance MACA [2] protocol was proposed to overcome the shortcoming of CSMA. The MACA used two control signal RTS and CTS for synchronization. If the packets transmitted by node are lost, the node used the binary exponential back off (BEB) algorithm to back off random interval of time before retrying. Using BEB the time starvation problem will occur; Reliability of Data is less in MACA. Overhead of control packets is more. The MACAW [3] uses more control packets for the synchronization such as RTS, CTS, ACK, DS and RRTS. The MACAW solve the MACA problem but in MACAW control overhead is more which is major challenge for mobile ad hoc networks. The FAMA [2], [11] which consist of both carrier sensing and collision avoidance dialog between the sender and intended receiver of packet. The control of channel is given one node at time and this node is guaranteed to transmit one or more packet to different destination without suffering from packet collisions. Carrier sensing by the sender will be done through the exchange of control packet RTS and CTS. The nodes which want transmit packet first require the channel before starting transmission. The Synchronization between sender and receiver require more control overheads. According to the BTMA [8], the channel is divided into the two parts one is called Data channel and the other is called Control channel. The Data channel is used for the data packet transmission while the control channel is used to transmit the busy tone signal. When a node want to transmit some Data packet it first sense the channel to check whether the busy tone is active if active then it reschedules the packet for transmission after some random rescheduling delay. If it is found not active then turn on the busy tone signal and start data transmission. According to BTMA no other node in the two hope neighborhood of the transmitting node is permitted to simultaneously transmit. So collision ratio is low in BTMA its bandwidth utilization is poor, transmission is blocked when one node occupies the cannel and other will wait when the channel will release, Control packets overhead is also more. DBTMA [8] is an extension of the BTMA scheme. In the DBTMA the transmission channel is also divided into two one is called control channel and the other is called the Data channel. Just like the BTMA the Data channel is used for data packets and the control channel is used for the control packets transmission (RTS-CTS) and also for transmitting busy tones. DBTMA will use the two busy tones on the control channel i.e. BTt and BTr. The BTt tone indicates that now the node is transmitting on the Data channel and the BTr indicates that it is now receiving Data on the Data channel. Bandwidth utilization is poor, control packets overhead is also more. The D-PRMA (Disturbed packet multiple access protocol) [10] is used for voice traffic support in ad hoc network it is the distributed scheme that can be used in ad hoc network. Basically DPRMA is a TDMA based protocol. The time division of channel is done into frame. The frame is further divided into slots and the slot is further divided into minislots and the minislots is further into two control fields, RTS/BI and CTS/BI as shown in figure 1 [10]. The BI show the busy indication when a ready node want a reserve a slot for transmission then these control fields are used for reservation and for overcoming hidden terminal problem. Starvation problem is also possible; when a slot is reserved for transmission other waits for release the slot. The SRMA/PA [11] was developed with the main objective of supporting both real time and non real time application in ad hoc wireless networks.

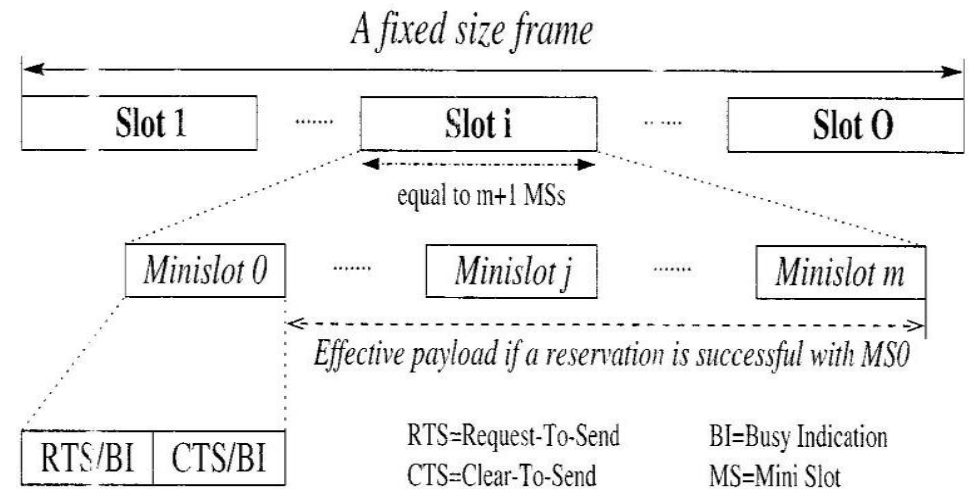

Figure 1: D-PRMA Frame division [10]. 
It is best suited for real time application because it uses priority assignment for traffic. Starvation problem also occur because the priority will be given to high priority traffic. Channel grap problem is possible. The FPRP [9] is a single channel time division multiple access (TDMA) based broadcast scheduling protocol. When a node requires a time slot, it uses the same contention mechanism as in SRMA/PA. FPRP is fully distributed and multiple reservations can be done at the same time. Time is divided into frames. Frames are two types reservation frame (RF) and information frame (IF). The RF frame is followed by a sequence of information frame (IF). When a node wants a reserve the channel then It will use the five phase dialogue for reservation. The main issues in FPRP are control overhead. The control overhead causes the poor bandwidth utilization. A Multichannel Reservation Multiple Access protocol for Mobile Ad Hoc Network (MRMA) was proposed by Kia Liu and Xiaoqin Xing [13]. The proposed protocol share the channel in efficient way it employs the RTS and CTS control packets on a common channel and select the free conflict channel for the Data transmission. After successful transmission it will used another control packet Ack replied to the sender on another common channel. The reservations overhead are more. Reservation Clash Handling to Optimize Bandwidth Utilization in MANETs is challenging task in Ad hoc networks.

The Extended R-CSMA protocol proposed by Ghalem Boudour and Zoubir Mammeri [14].Reservation process are complex it will used a lot of control overhead for channel reservation. Bandwidth Reservation for Heterogeneous Traffics in Mobile Ad hoc Networks (BRPHT) proposed by Ghalem Boudour and Zoubir Mammeri [15].

The proposed protocol multiplexes the several transmitting node on the same slot but each node allocated the required bandwidth so it provide more bandwidth to node.

BRPHT is based on the super frame structure. Collision chances are more due to transmitting several nodes on the same slot. Multi-Channel Medium Access Control with Hopping Reservation for Multi-hope Wireless Networks (MMAC-HR) proposed by Khaled Hatem Almotairi and Xuemin [16].

MMAC-HR optimizes the network performance. For the exposed terminal problem it will used the CSMA/CA over all channels. Using CSMA/CA on all channel will causes the energy expenditure and also the reservation overhead are more.

\section{Proposed Scheme}

In the proposed scheme for multichannel MAC protocol, the channel assignment is simple as compared to the previous multichannel MAC protocol channel assignment through reservation. In our proposed solution, we have divided the given channels channel into $\mathrm{N}$-sub channels and each sub channel is assigned an ID as shown in figure 2 (channel ID starts from 0 ).

\begin{tabular}{|c|}
\hline Channel ID-(N-1) \\
\hline \\
\hline \\
\hline \\
\hline Channel ID-2 \\
\hline Channel ID-1 \\
\hline Channel ID-0 \\
\hline
\end{tabular}

Figure 2: Division of Channel into N-sub channels

Each node in the network selects a particular channel for its transmission and can receive data at all channels. Channel selection is done using the following formula.

Node $_{\text {TX }}=\left(\right.$ Node $\left._{\text {id }}\right) \bmod \mathrm{N}$

Where Node ${ }_{\mathrm{TX}}$ is the channel used for transmission by Node $_{\mathrm{id}}$ and $\mathrm{N}$ is the total number of sub channels. According to the proposed scheme the channel will be assigned to the node according to the above formula. To select a transmission channel for a node, we divide by the No. of channels and remainder gives us the channel id for this particular node. For example, if the node ID on total sub channel such as Node id is 6 and the sub-channels are 5 then according to the formula the remainder is 1 so the channel id 1 will be used by node6 for transmission. The proposed scheme offers a simple and light weight mechanism for solving the problem of channel reservation in multi-channel MAC protocols. This scheme does not reserve a channel rather its selects a particular transmission channel for every node and that is used for ever by the respective node. The scheme not only eliminates the overhead involved in channel reservation but also reduces the no. of collisions between in the network by providing an efficient way of channel selection in a distributed fashion. Although the proposed scheme does not ensure (guarantee) that a distinct channel will be selected by neighboring nodes. In other words, it is possible that same channel will be selected by two neighboring nodes e.g. if node 6 and node 11 are neighbor nodes and we have 5 sub channels then the proposed scheme will result is selection of channel- ID (1) for both node 6 and node 11. As a result of using same transmission channel both cannot communicate at the same time otherwise it will result in collision. But in Ad hoc networks nodes are distributed randomly and the probability of having two nodes communicating at the same time, being neighbors and are assigned with same 
channel, is very low. According to Amdahl's law, we shall focus to optimize the part of a design that affects the performance at most. In other words, targeting to improve the fraction that rarely happens may results in less gain as compared to the overhead. Therefore we choose to use a simple (kind of random) scheme for channel selection. Remarkable improvement in the simulations results is observed by using proposed scheme. The major design question in our proposed scheme is that how many sub channels will be sufficient to achieve optimal results. Too many sub channels will result in under utilization as most of the channels will remain unused. On other hand, too few channels will results in selection of same channel by neighboring nodes and hence collisions. We have performed simulation with varying no. of sub channels ranging from 2 to 10 . We have found no more significant improvement in the results after 5 sub channels and we have reported results with 5 sub channels compared against single channel MAC protocol in section V. We have collected results various parameters such as Packet Delivery ratio, Throughput, Delay, Normalized Routing Load and Energy consumption. The Results show that the performance of the proposed protocol is best for the channel assignments according to new technique.

\section{Empirical Evaluations}

\section{A. Simulation Parameters}

We have compared the performance of the proposed scheme with traditional MAC protocol (single channel). For analysis, a testing environment had to be created in which the starting position and conditions are the same for all protocols. We have used NS-2 for simulation. Table I shows details of varying parameters used in simulation. Moreover, numbers of nodes in all simulations were 100, moving with uniform velocity of $5 \mathrm{msec}$ using Random waypoint mobility model, in square area of $1000 \times 1000 \mathrm{~m}^{2}$. CBR traffic sources (having packet interval of $0.2 \mathrm{sec}$ ) were used with UDP at transport layer and AODV at routing layer. For rest of the parameters ns-2 default settings were used.

\section{TABLE I}

VARYING SIMULATION PARAMETERS

\begin{tabular}{|l|l|l|}
\hline Simulation No. & No. of Flows & PKT Size \\
\hline 1 & 15 & 64 \\
\hline 2 & 15 & 128 \\
\hline 3 & 15 & 256 \\
\hline 4 & 15 & 512 \\
\hline 5 & 15 & 1024 \\
\hline 6 & 10 & 512 \\
\hline 7 & 20 & 512 \\
\hline 8 & 25 & 512 \\
\hline 9 & 30 & 512 \\
\hline
\end{tabular}

\section{B. Performance Metrics}

Now we define the performance Metrics which we used in the comparison of the protocols.

1.Throughput: The throughput can be defined as that how much information can be delivered from one location to another in given amount of time if we achieve the higher throughput the better because it implies that the protocols is delivering more information in given time. The throughput of a node can be defined as if the node sends the $\mathrm{x}$ number of bits in $\mathrm{t}$ amount of time then the throughput of this node may be defined as:

$\mathrm{Thr}=\mathrm{x} / \mathrm{t}$.

If it take the smaller amount of time and delivered more bits then the network achieve the maximum throughput. The maximum throughput is the ultimate objective of every network.

2. Success rate: The success rate means that how many packets successfully received by the destination node.

3. No. of Packets Delivered: It means that how many packets are delivered to the target node throughout the simulation. This parameter is useful in CBR traffic because CBR generates the packets with a fixed rate. The CBR ensure that the generation of packets remains same for all the protocol. Packets Delivery metrics gives a suggestion about the capability of the underlying protocol to transport the packets.

4. Delay: The Delay can be defined as how long take a time when the packet is sent from one location to another. If this value is minimum it means protocol is efficient and more desirable.

Delay (m sec): Average (Received Time - Sent Time)

5. Energy consumption: The energy consumption means that how many energy used by the node in the network. The protocol is a desirable if the energy consumption values is less. 
6. Packet delivery ratio (PDR): It is the ratio of the number of packets successfully received by the application layer of a destination node to the number of packets originated at the application layer of each node for that destination. The higher the PDR, the better, as it means that the protocol has less number of collisions. Packet delivery ratio means the number of packets received at the destination throughout the simulation. CBR generates the packets with a fixed rate so this metric is useful in CBR traffic. Thus the number of generated packets for the same simulation time remains the same for all protocols. This metric gives a direct idea about the ability of the underlying protocol to deliver the packets.

7. Normalized Routing Load (NRL): when the sender sends some data the NRL show ratio of the number of overhead (control packets) to the total number of data packets delivered. The NRL value is minimum the protocol is better. For more and more traffic coming into the network, it becomes harder for the network to manage all this traffic so it is called network load. This high network load or additional traffic on the network affects the MANET routing packets, which in result slows down the delivery of packets for reaching to the destination.

\section{Experiment And Results}

We compared the performance of proposed multi channel scheme with single channel MAC protocol. These set of experiments were performed to investigate the effect of packet size on various performance matrices such as PDR, Throughput, Energy used, Delay, and NRL. These Simulations are performed with packet size of 64, 128, 256, 512 and 1024 Bytes respectively. Figure 3 shows the comparative results of various metrics w.r.t to varying packet size. It is obvious that larger size packets will have longer airtime hence more collisions and longer delays, but at the same time protocols are delivering more bits on the same control overhead and same simulation time, due to this it will cause an increase in throughput and finally decrease in energy expenditure. The same behavior is depicted from single channel results. Multi channel results are best in all cases and mainly due to having very few collisions as nodes are communicating on different channels. While for single channel PDR is reduced when we increase the packet size because the larger packet will have loner air time. NRL and average delays are nearly unaffected with increase in packet size for our proposed multi channel scheme. Energy expenditure per node is increasing for multi-channel scheme with increase in packet size due to large amount of data transfer. Collectively, the result reveals that proposed multi channel scheme performs better with large packet size.

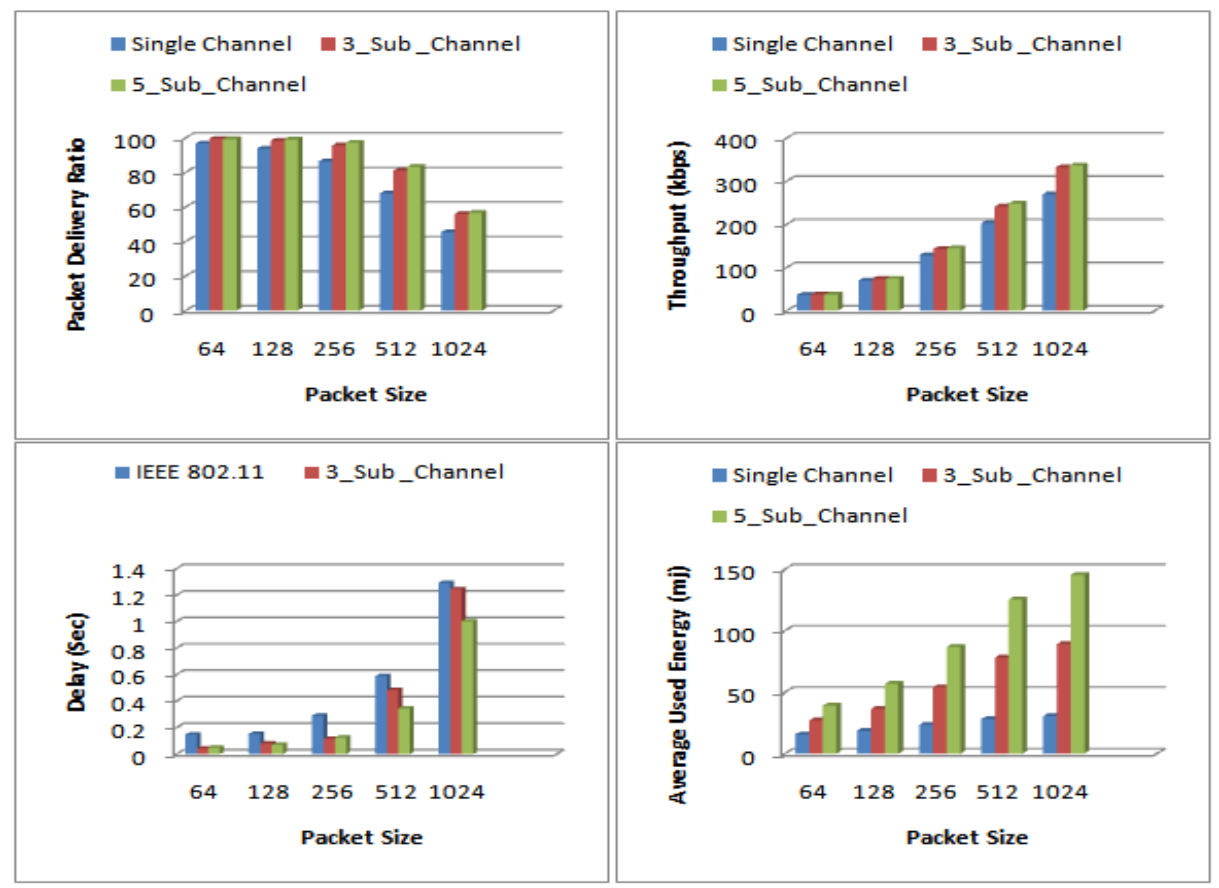

Next, we have analyzed the performance of the two schemes with varying no. of flows. The purpose of these experiments was to test the ability of the both protocols to adopt itself to varying conditions and under varying stresses. This experiment helps us in understanding the maximum possible upper limit of these MAC protocols. Performance of the protocols is evaluated with flows 5, 10, 15, 20, 25 and 30. Figure 4 show the comparative results of the single and proposed multi channel MAC Protocols scheme w.r.t to various number of flows to test their performance in congested environment. The relatively low PDR of single channel MAC protocol is because of its aggressive reaction on link failure which results in failure of other established routes 
due to collision. Increasing the number of flows then congestion will occurs in single channel MAC protocol that will ultimately lead toward collisions due to which links failure will occur and the protocol will try to discover the new link for transmission. So the Routing packet ratio increased to discover the new link. The Average Delay of Single Channel MAC protocol is increased with the increased in flows as from figure 4, this is because of the congestion in the network. Collectively, the result reveals that proposed multi channel scheme performs better with various flows in the congested networks.

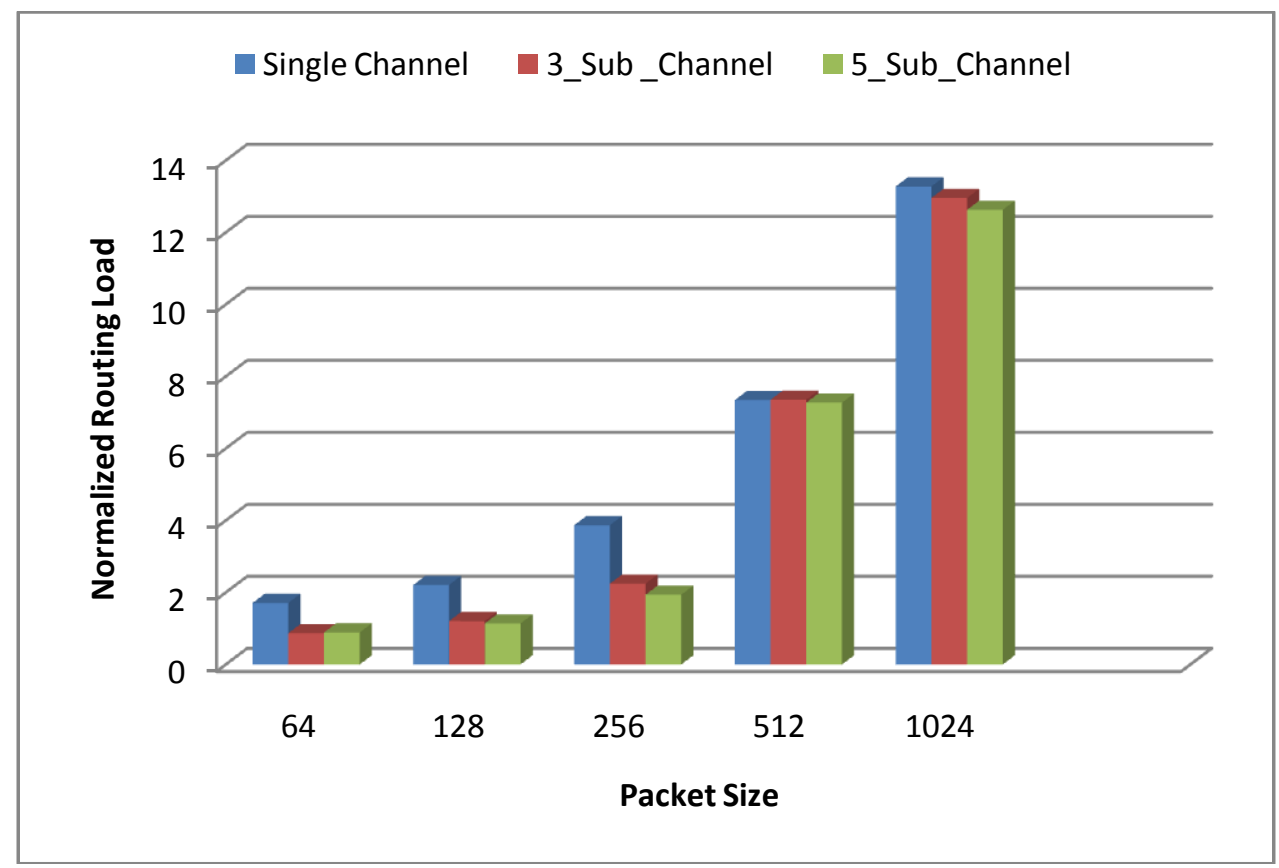

Figure 3: Various PKT Size
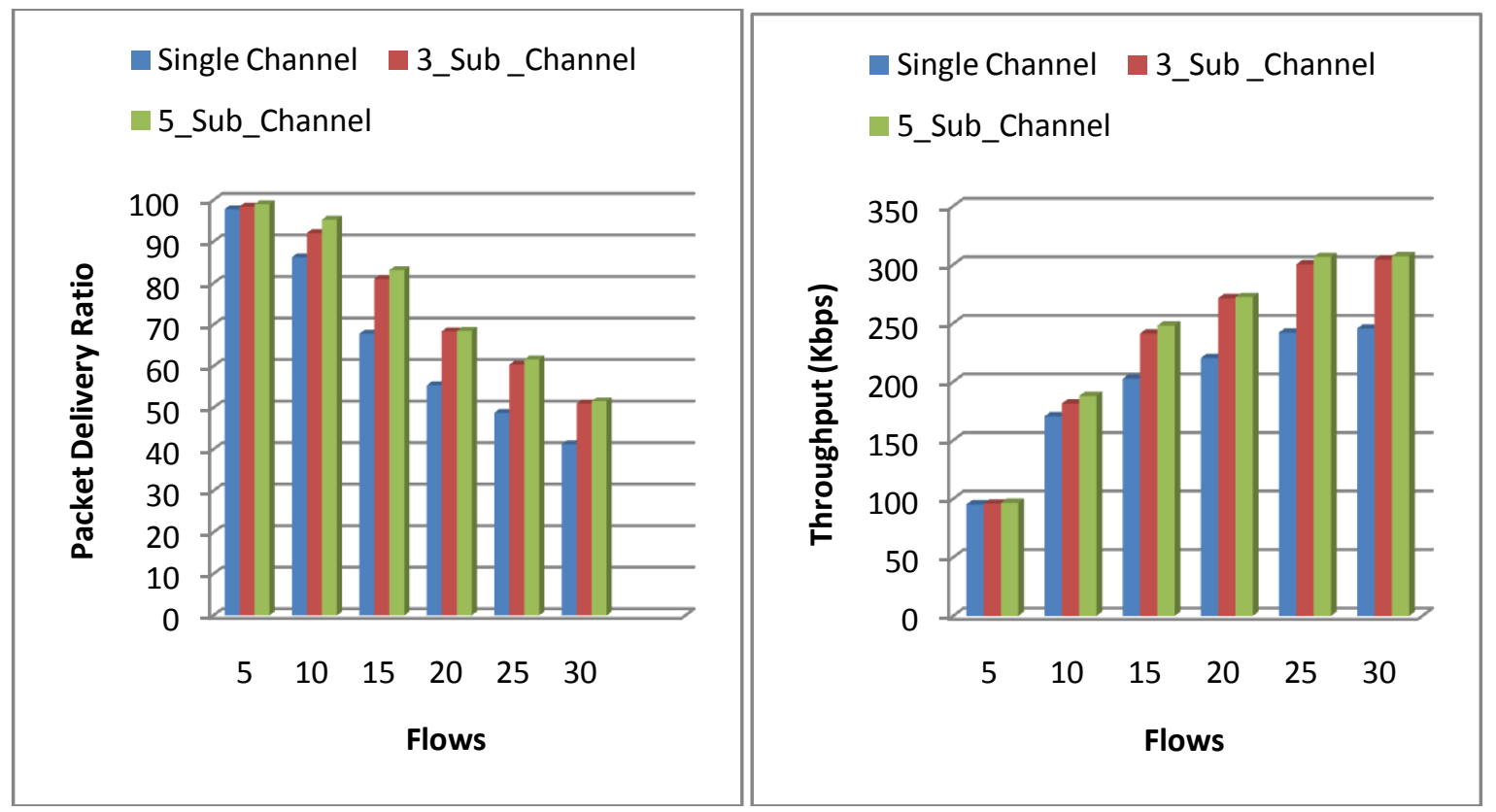

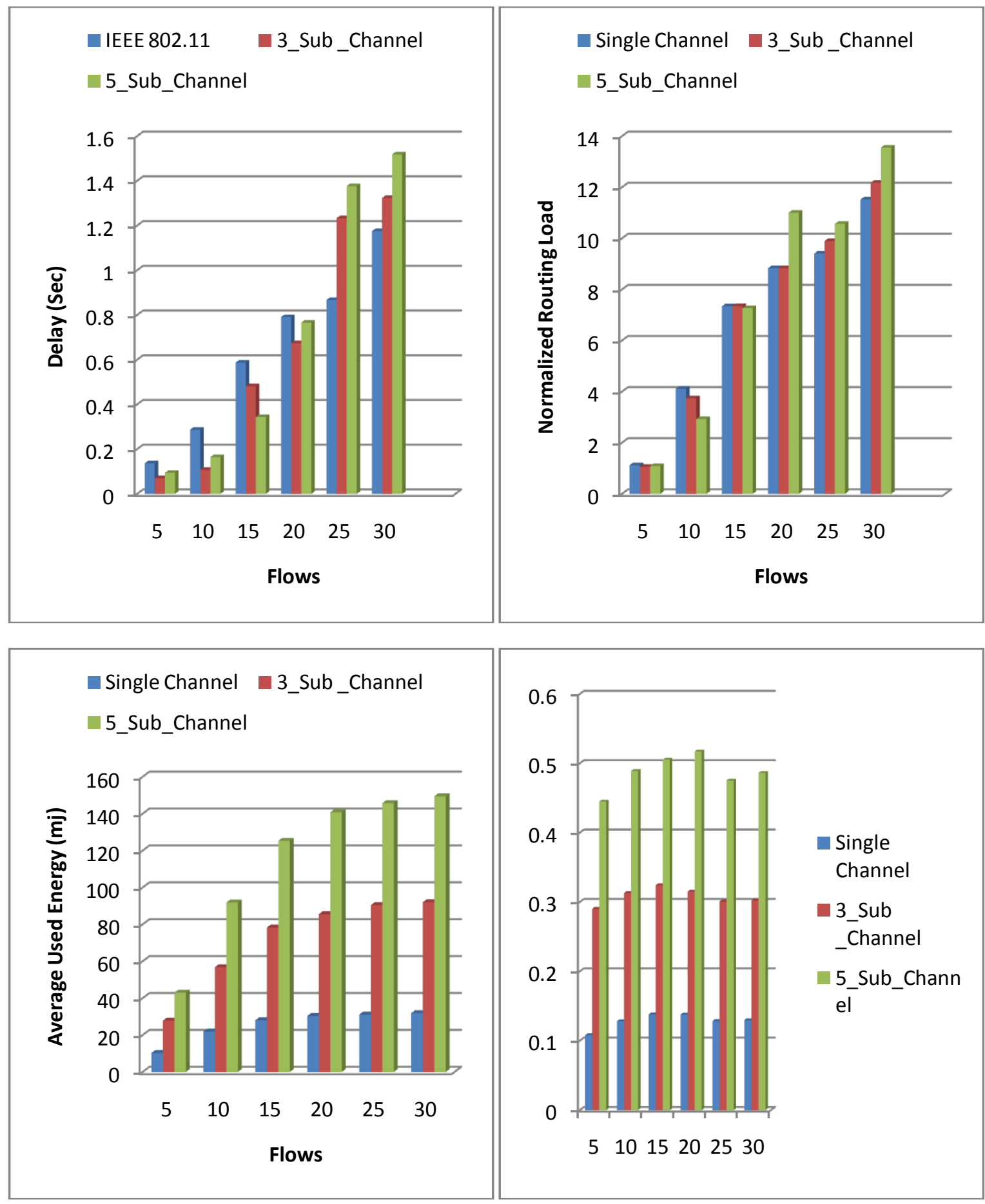

Figure 4: Various Number of flows

\section{Conclusions And Future Directions}

In this paper, a Multi Channel Scheme has been proposed for mobile Ad hoc networks. The proposed scheme offers a simple and light weight mechanism for solving the problem of channel reservation in multichannel MAC protocols. The key feature of our technique is the Zero control overhead, reduced collision and effective channel utilization. The performance of the Single channel has been compared with that of the proposed Multi channel scheme under various parameters in the simulation environment NS-2. It is observed that the performance of proposed multi channel scheme outperform then Single Channel MAC protocol. From all these experiments, it is concluded that proposed Multi Channel scheme provides the valuable solution for mobile ad hoc networks. We are looking forward to extend the current work in two directions. First, although our proposed scheme has improved the performance of traditional MAC protocol but, its real gain will be 
revealed when compared against some existing multi channel protocols. As ns-2 code of any multi channel protocol is not available, so we will implement some selected multi channel protocols for comprehensive analysis. Secondly, proposed scheme channel assignment is based on a simple formula that does not guarantee selection of a distinct channel for neighboring nodes. We need some how to modify the scheme to overcome its inherited problem.

\section{References}

[1] Blerta Bishaj, MAC protocols, contention-based with reservation and scheduling, Helsinki University of Technology 2008.

[2] C.Siva Ram Murthy and B.S Manjo, Ad hoc Wireless Network Architecture and protocols, 2nd Edition, Published by Dorling Kindersley (India).

[3] V. Bharghavan, A. Demers, S. Shenker, and L. Zhang,"MACAW: A media access protocol for wireless LAN's", In SIGCOMM '94, pages 212-225. ACM, 1994.

[4] N.Abramson,"TheALOHA system-another alternative for computer communication," Proc.of AFIPS FJCC, pp-281-285, 1970.

[5] Mariam Kaynia, Geir E.ien, Nihar Jindal, David Gesbert," Comparative Performance Evaluation of MAC Protocols in Ad Hoc Networks with Bandwidth Partitioning”, Dept. of Electrical and Computer Engineering, University of Minnesota, USA, 2009.

[6] WenHwa Liao and WenChin Chung," An Efficient Multi-Channel MAC Protocol for Mobile Ad Hoc Networks", Department of Information Management Tatung University, Taiwan, 2009 International Conference on Communications and Mobile Computing.

[7] ChihMin Chao1, HsienChen Tsai, and Kuan-Ju Huang," A New Channel Hopping MAC Protocol for Mobile Ad Hoc Networks", Department of Computer Science and Engineering National Taiwan Ocean University, Taiwan, IEEE 2009.

[8] Zygmunt J. Haas and Jing Deng,"Dual Busy Tone Multiple Access (DBTMA)-a Multiple Access Control Scheme for Ad Hoc Networks" IEEE TRANSACTIONS ON COMMUNICATIONS, VOL. 50, NO. 6, JUNE 2002.

[9] C. L. Fullmer and J. Garcia-Luna-Aceves,"FAMA-PJ: A channel access protocol for wireless LANs. In Mobile computing and Networking", Berkley, CA (USA), pages 76-85. ACM, November 1995.

[10] Shengming Jiang, Jianqiang Rao, Dajiang He and Xinhua Ling,"A Simple Distributed PRMA for MANETs, IEEE TRANSACTIONS ON VEHICULAR TECHNOLOGY, VOL. 51, NO. 2 MARCH 2002.

[11] Ritun Patney,"Survey on Performance Evaluation Techniques for Medium Access Control Protocols", Washington University in St. Louis 2007.

[12] Zhenyu Tang and J. J. Garcia-Luna-Aceves,"Hop-Reservation Multiple Access (HRMA) for Ad-Hoc Networks", Computer Engineering Department School of Engineering University of California, IEEE 1999.

[13] Kai Liu and Xiaoqin Xing: A Multichannel Reservation Multiple Access Protocol for Mobile Ad hoc Networks: ICC 2008.

[14] Ghalem Boudour and Zoubir Mammeri: Reservation Clash Handling to optimize Bandwidth Utilization in MANETs: International Conference on Communication Theory, Reliability, and Quality of Services 2008.

[15] Ghalem Boudour and Zoubir Mammeri: Bandwidth Reservation for Heterogeneous Traffics in Mobile Wireless Networks: Fifth Advanced International Conference on Telecommunications 2009.

[16] Khaled Hatem Almotairi and Xuemin: Multi-channel Medium Access Control with Hopping Reservation for Multi-hop Wireless Networks in IEEE Globecom 2010. 